\title{
Biodistribution, kinetics, and biological fate of SPION microbubbles in the rat
}

This article was published in the following Dove Press journal:

International Journal of Nanomedicine

23 August 2013

Number of times this article has been viewed

\author{
Åsa Barrefelt ${ }^{1,2, *}$ \\ Maryam Saghafian ${ }^{2, *}$ \\ Raoul Kuiper ${ }^{3}$ \\ $\mathrm{Fei} \mathrm{Ye}^{4}$ \\ Gabriella Egri ${ }^{5}$ \\ Moritz Klickermann ${ }^{5}$ \\ Torkel B Brismar' \\ Peter Aspelin' \\ Mamoun Muhammed ${ }^{4}$ \\ Lars Dähne ${ }^{5}$ \\ Moustapha Hassan ${ }^{2,6}$ \\ 'Department of Clinical Science, \\ Intervention and Technology, Division \\ of Medical Imaging and Technology, \\ Karolinska Institutet, and Department \\ of Radiology, Karolinska University \\ Hospital-Huddinge, Stockholm, Sweden; \\ ${ }^{2}$ Experimental Cancer Medicine, \\ Department of Laboratory Medicine, \\ Karolinska Institutet, Stockholm, \\ Sweden; ${ }^{3}$ Karolinska Institute Core \\ Facility for Morphologic Phenotype \\ Analysis, Clinical Research Center, \\ Karolinska University Hospital- \\ Huddinge, Stockholm, Sweden; \\ ${ }^{4}$ Division of Functional Materials, \\ Department of Materials and Nano \\ Physics, Royal Institute of Technology, \\ Stockholm, Sweden; ${ }^{5}$ Surflay Nanotec \\ $\mathrm{GmbH}$, Berlin, Germany; ${ }^{6} \mathrm{Clinical}$ \\ Research Center, Karolinska University \\ Hospital-Huddinge, Stockholm, Sweden \\ *These authors contributed equally to \\ this work
}

Correspondence: Moustapha Hassan Experimental Cancer Medicine, Clinical Research Center, Karolinska University Hospital Huddinge,

SE-I4I 86 Stockholm, Sweden

Tel +468 58583862

Fax +468 58583800

Email moustapha.hassan@ki.se
Background: In the present investigation, we studied the kinetics and biodistribution of a contrast agent consisting of poly(vinyl alcohol) (PVA) microbubbles containing superparamagnetic iron oxide (SPION) trapped between the PVA layers (SPION microbubbles).

Methods: The biological fate of SPION microbubbles was determined in Sprague-Dawley rats after intravenous administration. Biodistribution and elimination of the microbubbles were studied in rats using magnetic resonance imaging for a period of 6 weeks. The rats were sacrificed and perfusion-fixated at different time points. The magnetic resonance imaging results obtained were compared with histopathologic findings in different organs.

Results: SPION microbubbles could be detected in the liver using magnetic resonance imaging as early as 10 minutes post injection. The maximum signal was detected between 24 hours and one week post injection. Histopathology showed the presence of clustered SPION microbubbles predominantly in the lungs from the first time point investigated (10 minutes). The frequency of microbubbles declined in the pulmonary vasculature and increased in pulmonary, hepatic, and splenic macrophages over time, resulting in a relative shift from the lungs to the spleen and liver. Meanwhile, macrophages showed increasing signs of cytoplasmic iron accumulation, initially in the lungs, then followed by other organs.

Conclusion: The present investigation highlights the biological behavior of SPION microbubbles, including organ distribution over time and indications for biodegradation. The present results are essential for developing SPION microbubbles as a potential contrast agent and/or a drug delivery vehicle for specific organs. Such a vehicle will facilitate the use of multimodality imaging techniques, including ultrasound, magnetic resonance imaging, and single positron emission computed tomography, and hence improve diagnostics, therapy, and the ability to monitor the efficacy of treatment.

Keywords: biodistribution, microbubbles, superparamagnetic iron oxide, pharmacokinetics, magnetic resonance imaging, histopathology

\section{Introduction}

The increasing number of newly developed nanodevices, nanoparticles, and multifunctional carriers for biomedical application highlights the importance of thorough preclinical investigation of their toxicity, biodistribution, pharmacokinetics, and clearance, as well as their interaction with host tissues. Several investigations utilizing in vivo imaging and histopathologic surveys are presently being conducted to determine the biological properties, biodistribution, and toxicity of different drug carriers or nanodevices. ${ }^{1-3}$ Potentially suitable nanocarriers are widely variable, ranging from nanoscale biologically derived or artificial virus-like particles to engineered (up to micron-sized) particles; consequently, they have a highly variable biological impact 
on the target species. For instance, Singh et $\mathrm{al}^{2}$ studied a plant virus, ie, cowpea mosaic, that is used for the ability of its coating proteins to attach to a variety of molecules by means of genetic modifications of the virus. These authors showed that cowpea mosaic is relatively safe and nontoxic, but also that it could cause leukopenia at high doses. Silica nanoparticles are an example of a nonbiological vehicle used for drug delivery and hybridization. Using ${ }^{125}$ I-radiolabeled silica nanoparticles and histologic survey, Xie et $\mathrm{al}^{3}$ found accumulation in the lungs, liver, and spleen of mice due to uptake by macrophages. The authors suggest that uptake of silica nanoparticles by macrophages and subsequent activation might increase the risk of liver injury in mice. Preclinical studies are of great importance in evaluating the potential toxicity and adverse effects of nanoparticles prior to clinical investigations.

Superparamagnetic iron oxide nanoparticles (SPIONs) have previously been reported in the literature for several nanomedicine applications. One example is the use of hydrotropic magnetic micelles for combined magnetic resonance (MR) imaging and cancer treatment, ${ }^{4-6}$ chemotherapy, ${ }^{7}$ and targeting of hepatocytes using SPION-loaded chitosan-linoleic acid. ${ }^{8}$ They can also be used for delivery of drugs such as doxorubicin, platinum compounds, anti-inflammatory drugs, and prodrugs. ${ }^{9-12}$ SPIONs are interesting nanoparticles with clinical potential both in the imaging field and for drug delivery.

Small injectable particles have also been shown to be of use as a diagnostic aid to improve the visibility of internal body structures, enhance the contrast between normal and diseased tissue, visualize abnormalities in tissue, and follow the progress of disease. ${ }^{13-20}$ For instance, SPION nanoclusters have been used as a negative contrast agent in liver and heart imaging, ${ }^{21}$ and SPION-folate-poly(ethylene glycol) has been used for lung cancer imaging. ${ }^{22}$ The microbubbles used in this investigation represent a novel microdevice consisting of poly(vinyl alcohol) (PVA) containing SPION in between the shells. SPION microbubbles are designed to function as a contrast-enhancing device for ultrasound imaging and also as a contrast-enhancing agent for MR. The PVA shell has the capacity to be functionalized by several ligands for further attachment of radioactive tracers such as ${ }^{99} \mathrm{~m}$ Tc or antibodies. Thus, the microbubbles are of interest as a multifunctional microdevice for targeted drug delivery and multimodal imaging. At present, information on tissue distribution and toxicity and/or adverse effects of the particles exceeding nanoscale is limited.

In a previous study using multimodal imaging, single photon emission computed tomography (SPECT)/ computed tomography/MR imaging, we showed that SPION microbubbles are mainly distributed to the lungs and liver in the rat and are slowly eliminated. ${ }^{23}$ In the current study, we further investigated the biological fate and kinetics of SPION SPECT in different tissues. We utilized a histopathologic survey to compare, correlate, and confirm the results obtained from MR examination. MR imaging is a noninvasive approach for measuring and following small changes in the same animals over time. This technique may help in reducing the number of animals, decreasing intraindividual variation, and refining the experiments, which may enhance the statistical power of the experiment. ${ }^{24-26}$

\section{Materials and methods Synthesis of microbubbles containing SPION}

The SPION microbubbles used in the current study were synthesized and produced as described previously. Briefly, PVA was dissolved in water $(20 \mathrm{~g} / \mathrm{L})$. Next, $7.6 \mathrm{~g}$ of sodium periodate was added and the mixture was incubated at $85^{\circ} \mathrm{C}$ for one hour, cooled to room temperature, then suspended for 3 hours using an Ultra Turrax (IKA-2000, Staufen, Germany) at 8,000 rpm followed by washing three times in a cream centrifugal separator (RZ OPS, 3,000 rpm, Merryfarm, Kiev, Ukraine). The microbubbles were then modified with aminoguanidine to enable further coupling with different layers according to the following procedure: the microbubbles $\left(100 \mathrm{~mL}\right.$, approximately $7.1 \times 10^{10}$ microbubbles) were washed in $0.1 \mathrm{M} \mathrm{HEPES}$ buffer at $\mathrm{pH}$ $8(200 \mathrm{~mL})$, aminoguanidine $(5 \mathrm{mg} / \mathrm{mL})$ was added, and the suspension was put on a shaker for 3 days followed by washing and centrifugation.

\section{Grounding using the layer-by-layer method}

Following aminoguanidine grounding, the microbubbles were coated using the layer-by-layer technique ${ }^{27}$ and functionalized using different layers of polystyrene sulfonate $70,000 /$ polyethyleneimine 25,000 /polystyrene sulfonate 70,000 /polyethyleneimine 25,000 /polystyrene sulfonate 70,000 . The coating solutions were $2 \mathrm{~g} / \mathrm{L}$ in $50 \mathrm{mM}$ acetate buffer, $\mathrm{pH} 5.6$, and $0.2 \mathrm{M} \mathrm{NaCl}$. After each layer was added, the microbubbles were washed four times, the upper phase was separated by centrifugation $(30 \times \mathrm{g}, 40$ minutes $)$, and the subnatant was removed. This was followed by addition of the next layer. ${ }^{27}$ 


\section{Coating with magnetite (iron oxide)}

A poly(allyl) amine hydrochloride (PAH, 15,000) layer was attached to the microbubbles to undercoat the magnetite. The coating solution contains $1 \mathrm{~g} \mathrm{PAH} / \mathrm{L}$ in a $50 \mathrm{mM}$ acetate buffer (pH 5.6) plus $0.1 \mathrm{M} \mathrm{NaCl}$. The aqueous magnetite coating solution was added to the microbubbles $\left(12 \mathrm{~mL} / 10^{9}\right.$ microbubbles) plus $1 \mathrm{M}$ acetate buffer (pH 5.6) to give an acetate concentration of $50 \mathrm{mM} . \mathrm{NaCl}(1 \mathrm{M})$ was added dropwise until the $\mathrm{NaCl}$ concentration reached $0.1 \mathrm{M}$. The sample was put on a shaker at room temperature overnight, the upper phase was separated by centrifugation $(30 \times \mathrm{g}$, 40 minutes), and the subnatant was removed. When the first magnetic layer had been attached to the microbubbles, they were washed and separated in a magnetic field; thus, no further centrifugation was necessary. This procedure was repeated for each layer. The microbubbles (with the layers: magnetic layer (Mag1)/PAH1/Mag2/PAH2/Mag3/ PAH3) were coated by $(2 \times 80 \mathrm{~mL}$ polystyrene sulfonate $)$ per $5 \times 10^{10}$ microbubbles as described above. PAH was labeled with fluorescein isothiocyanate at $\mathrm{pH} 8$ overnight. The product was purified by dialysis. The negatively charged microbubbles with polystyrene sulfonate as the outmost layer were then coated with the fluorescein-labeled positively charged PAH. Thereafter, the microbubbles were again coated with polystyrene sulfonate to provide a negatively charged surface.

\section{Characterization of SPION microbubbles}

The microbubbles were analyzed by bright field and fluorescent microscopy (image size $550 \mu \mathrm{m} \times 550 \mu \mathrm{m}$, Leica TCS SPE, Wetzlar, Germany). The morphology of the SPION microbubbles was characterized using field emission transmission electron microscopy (TEM, JEM-2100F, JEOL, Tokyo, Japan). The TEM sample was prepared by depositing an aqueous suspension of SPION microbubbles onto a copper grid covered with a carbon film. The grid was dried, loaded in the sample holder, and examined under TEM operating at an accelerating voltage of 200 $\mathrm{kV}$. The hydrodynamic size of the SPION microbubbles was measured using a flow cytometer (Partec CyFlow, Partec GmbH, Münster, Germany). Forward scatter as well as sideward scatter was measured to determine the size and surface area of the microbubbles. Moreover, three fluorescent lasers of different wave lengths (FL1 [536 $\pm 40 \mathrm{~nm}]$, FL2 $[590 \pm 50 \mathrm{~nm}]$, and FL3 [675 nm]) were applied to the microbubble solution to give information as to whether the dye was homogenously distributed among the microbubbles.

\section{Animal studies}

The study was approved by the Stockholm Southern Ethical Committee on Animal Research and was performed in accordance with Swedish Animal Welfare law. Male Sprague-Dawley rats weighing $250 \pm 50 \mathrm{~g}$ were purchased from Charles River (Charles River Laboratories, Sulzfeld, Germany). Upon arrival, the animals were allowed to acclimatize for at least one week before the start of the experiment. The animals had access to food and water ad libitum, and were kept at a facility with a 12-hour light/dark cycle, controlled humidity $(55 \% \pm 5 \%)$, and controlled temperature $\left(21^{\circ} \mathrm{C} \pm 2{ }^{\circ} \mathrm{C}\right)$.

The rats were anesthetized using an intraperitoneal injection of $60 \mathrm{mg} / \mathrm{kg}$ sodium pentobarbital (APL, Kungens Kurva, Sweden). The rats were injected intravenously with SPION microbubbles $\left(0.6 \mathrm{~mL}\right.$ of $0.5 \times 10^{9}$ microbubbles per $\left.\mathrm{mL}\right)$ via the tail vein.

\section{MR imaging and pharmacokinetic modeling}

Three rats were imaged using a commercially available 3 Tesla MR scanner (Siemens Trio, Siemens, Erlangen, Germany). The rats were anesthetized, placed head first in the prone position in an extremity coil, and imaged in the coronal plane at 3 Tesla. The rats were then injected with SPION microbubbles and imaged for 2 hours. Repeat imaging was performed at $3,6,12,24$, and 48 hours and at one, $2,3,4,5$, and 6 weeks. A gradient-echo T2* sequence with a repetition time of $2,000 \mathrm{msec}$, field of view of $148 \times 250 \mathrm{~mm}$, slice thickness of $3 \mathrm{~mm}$, and 12 stepwise increasing echo times of 2-22.9 msec was used to scan the rats using one or two signal averages. The duration of the one signal average T2* sequence was 3.5 minutes, and the two signal average sequence had a duration of 7.4 minutes. The signal intensity was measured manually by placing $10-40 \mathrm{~mm}^{2}$ circular regions of interest on the images with different echo times. In the liver, full transaxial relaxation occurred very quickly; therefore, the calculations were based on fewer echo times (2-11.5 msec) in order to prevent underestimation. ${ }^{23} \mathrm{~T} 2 *$ was calculated as the slope of a semilog plot of the signal intensities versus echo times. The signal intensity was used as an indicator for SPION concentrations in the liver tissue. The elimination half-life $\left(\mathrm{t}^{1} / 2\right)$ was calculated from pharmacokinetic modeling based on R2* $\left(\mathrm{ms}^{-1}\right)$ versus time (hours) The modeling was performed and the curve was fitted to a one-compartment open model using WinNonLin version 5.0 (Pharsight, Mountain View, CA, USA). The slope of the terminal elimination phase was used to calculate the half-life. 
To exclude the effects of the magnetic field from the MR scanner on SPION microbubbles, four additional rats were injected with SPION microbubbles. Two animals were scanned repeatedly by 3 Tesla MR imaging over one week and two animals served as controls (without scanning). All four rats were euthanized, perfused, and investigated histologically one week (168 hours) post injection.

\section{Necropsy and histology}

To verify findings on MR imaging, rats were injected and anesthetized as described above. At 10 minutes, one, 3, 6, 12, 24, and 48 hours, as well as one, $2,3,4$, and 6 weeks post injection, three rats per time point were anesthetized by intraperitoneal injection of $60 \mathrm{mg} / \mathrm{kg}$ sodium pentobarbital and fixed onto a surgical pad in the supine position. A surgical incision along the chest midline was made, and the skin, the anterior part of the peritoneum, and the diaphragm were cut. The right atrium was incised to release intravascular pressure, after which $40 \mathrm{~mL}$ of heparinized phosphate-buffered saline and $60 \mathrm{~mL}$ of ice-cold fixation solution (paraformaldehyde 4\%, Solveco, Rosersberg, Sweden) were injected slowly through a needle inserted into the left ventricle. The perfusion of the corporal circulation was monitored by observing the liver turning pale. Tissues were then dissected and transferred to paraformaldehyde $4 \%$. After 48 hours, the paraformaldehyde was replaced by $70 \%$ ethanol before further dehydration and paraffinization of selected tissue samples in a vacuum infiltration processor. Lungs, liver, spleen, adrenal glands, kidneys, brain, eyes and Harderian glands, inguinal and mesenteric lymph nodes, as well as the sternum were embedded in paraffin according to RENI trimming guidelines. ${ }^{28}$ Sections $(4 \mu \mathrm{m})$ were mounted on superfrost glass slides and stained with hematoxylin and eosin and Perl's Prussian blue staining for iron. Unstained sections were used to verify remaining fluorescence of fluorescein isothiocyanate-labeled microbubbles in tissue sections. In addition, immunohistochemistry was performed with a primary antibody against CD68 macrophage marker (MCA 341, AbD Serotec, Kidlington, UK) in order to confirm phagocytosis of microbubbles by macrophages in the lungs, liver, and spleen. The secondary antibody was alkaline phosphatase-conjugated rabbit anti-mouse (A4312, Sigma, St Louis, MO, USA), and Vector red substrate (SK5100, Vector Laboratories Inc, Burlingame, CA, USA) was used to produce a red signal for bright field as well as red fluorescent microscopy according to the manufacturer's protocol. Slides were coverslipped utilizing 4',6-diamidino2-phenylindole (DAPI) containing mounting medium (Vector Vectashield ${ }^{\circledR}$ H1200, Vector Laboratories Inc) to produce a nuclear counterstain. The slides were evaluated using an A1R confocal microscope (Nikon Corporation, Tokyo, Japan) with appropriate fluorescence filters for fluorescein isothiocyanate, Cy5, and DAPI.

For histologic quantification of microbubbles in tissues, Perl's Prussian blue stained slides were digitally scanned using a Pannoramic slide scanner (3DHistech, Budapest, Hungary). Repeated measurement of a minimum area of $1 \mathrm{~mm}^{2}$ was selected for lungs, liver, and spleen $(\mathrm{n}=5)$ from each animal for digital counting of microbubbles based on their iron content, using Elements BR software (Nikon Instruments Inc, Melville, NY, USA).

\section{Results \\ Physical properties of SPION microbubbles}

SPION microbubbles did not show self-aggregation in water-based suspension, nor did the suspension contain any debris as judged by visual inspection. The use of bright field in combination with fluorescent microscopy showed that the SPION microbubbles could be labeled efficiently with fluorescein isothiocyanate (Figure 1A and B). High resolution TEM revealed that the SPION microbubbles had a diameter of approximately $3.1 \mu \mathrm{m}$ (Figure 1C). Single crystal structures of SPION embedded in between the shells of SPION

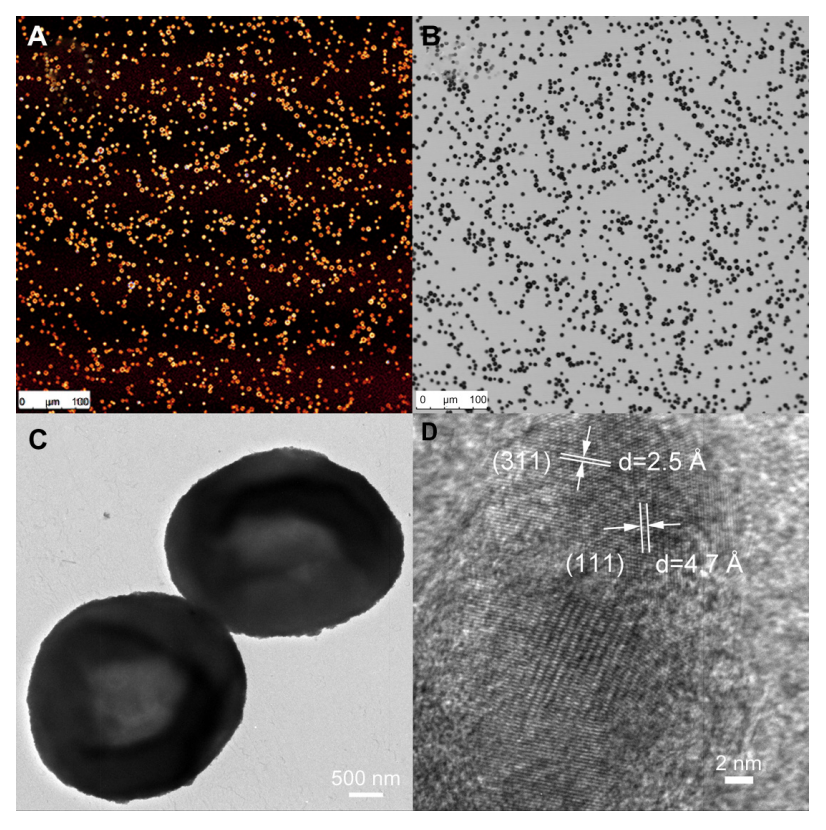

Figure I Properties of SPION microbubbles. SPION microbubbles seen under light microscopy (image size $550 \mu \mathrm{m} \times 550 \mu \mathrm{m}$ ) using the fluorescein isothiocyanate channel (A) and in bright-field microscopy setting (B). Two microbubbles seen on electron microscopy (C) and crystal lattice parameters of SPION (D) using field emission transmission electron microscopy.

Abbreviation: SPION, superparamagnetic iron oxide. 
microbubbles had a size of $12.4 \mathrm{~nm}$ (Figure 1D). Consistent with these observations, flow cytometry verified that SPION microbubbles were not aggregated and did not contain any debris (Figure 2A-C). Moreover, forward scatter showed a narrow size distribution among the microbubbles, while the results from fluorescent lasers at different wave lengths showed a homogenous dye distribution among the microbubbles (Figure 2D-F). In addition to the aforementioned physical properties, we found that the microbubbles could be visualized in tissues by bright field microscopy when using polarized light. Figure $3 \mathrm{~A}$ shows intravascular SPION microbubbles in the lungs in the form of single circulating structures as well as intravascular aggregates at 10 minutes post injection, identified by their birefringent walls. Generally, birefringence did not decline over time, except for a minor number of phagocytosed microbubbles at time points 3 weeks and later which showed a mild decrease in birefringence (Figure 3B).

\section{In vivo $M R$ results}

Dynamic three-dimensional MR imaging shows T2* weighted images of a rat prior to (Figure 4A) and at the investigated time points post injection (Figure 4B-I). Prior to injection of SPION microbubbles, the liver appears white on the $\mathrm{T} 2 *$ weighted image (Figure $4 \mathrm{~A}$ ) followed by a rapid change in signal intensity. A signal decrease was observed in the liver as early as 10 minutes post injection, indicating the presence of iron due to accumulation of SPION microbubbles (Figure 4B). The signal significantly decreased from one hour (Figure 4C) to 24 hours post injection (Figure 4D), remained low at 48 hours (Figure 4E), and reached its minimum at one week (Figure 4F). A continuous increase in signal intensity was observed from 2 weeks post injection (Figure 4G). A marked decrease was seen from 4 weeks post injection (Figure 4H), reaching nearly normal levels (pre injection) at 6 weeks post injection (Figure 4I). Figure 5 shows a pharmacokinetic curve of R2* $\left(\mathrm{s}^{-1}\right)$ versus time (hours) in the liver. The minimum signal that corresponds to a maximum concentration was reached at one week post injection (Figure 5). The half-life of SPION microbubbles in the liver was calculated to be 31 days $\left(\mathrm{t}_{1 / 2} 741 \pm 219\right.$ hours $)$.

\section{Histology}

Figure 6 shows results from microscopic examination of lungs (Figure 6A-D), liver (Figure 6E-H), and spleen (Figure 6I-L). Clustered SPION microbubbles were observed in the above mentioned organs from the first time point investigated (10 minutes post injection). Other tissues (brain, kidney, adrenal glands, mesenteric and inguinal lymph
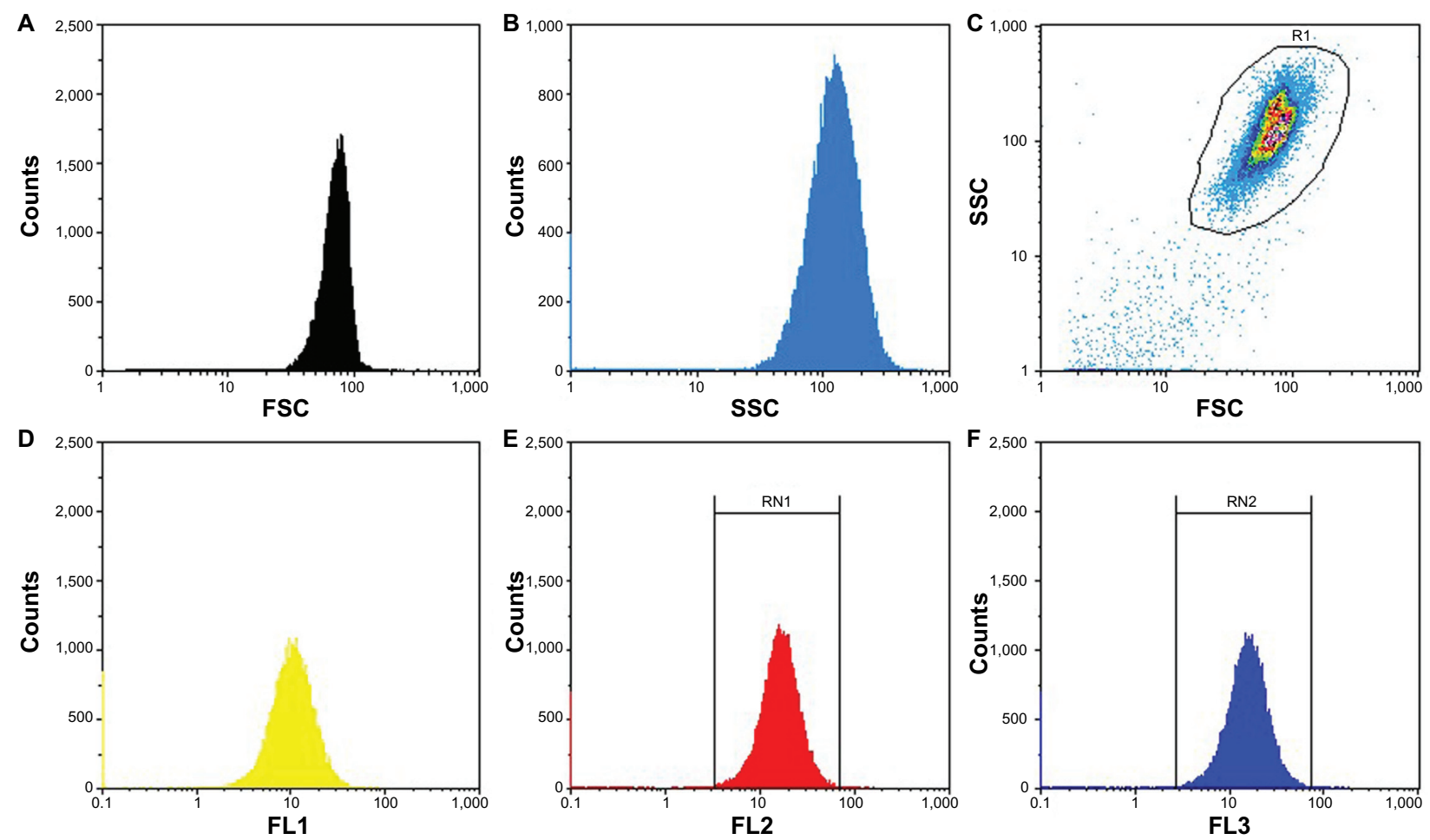

Figure 2 Flow cytometric analysis of SPION microbubbles. FSC shows size distribution (A and $\mathbf{C}$ ) and SSC provides information about the surface (B and C), indicating a narrow size distribution and homogeneous surface. The different fluorescent lasers [FLI (D) $536 \pm 40 \mathrm{~nm}$; FL2 (E) $590 \pm 50 \mathrm{~nm}$; FL3 (F) $675 \mathrm{~nm}$ ] show that the dye is homogenously distributed among the SPION microbubbles.

Abbreviations: FSC, forward scattering; SSC, sideward scattering; SPION, superparamagnetic iron oxide. 


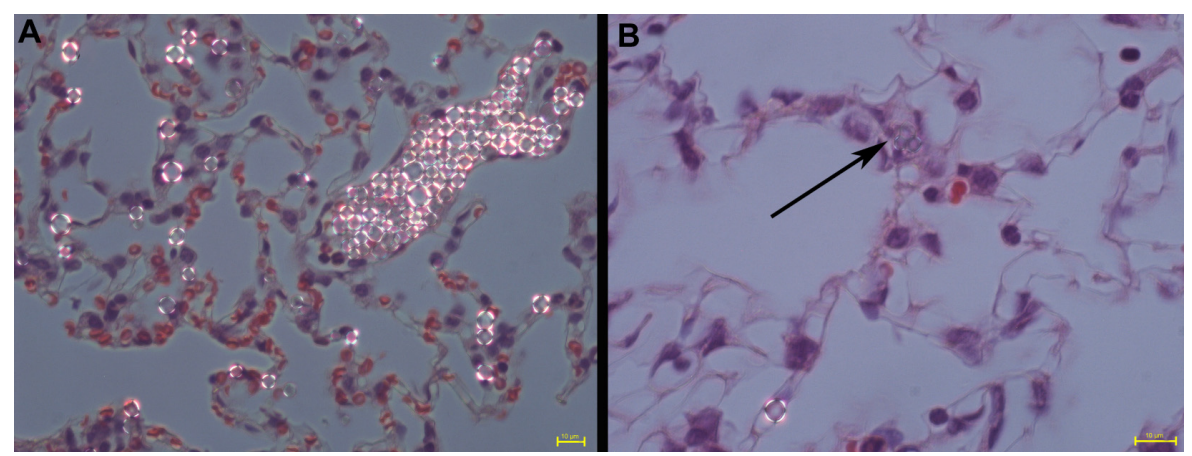

Figure 3 Polarizing properties of SPION microbubbles. Single and clustered SPION microbubbles in pulmonary vasculature and macrophages (I0 minutes post injection) show birefringent walls when viewed using polarized light (A). At 3 weeks post injection, a decrease in birefringence is shown in phagocytosed SPION microbubbles [arrow, (B)]. The size bar represents $10 \mu \mathrm{m}$.

Abbreviation: SPION, superparamagnetic iron oxide.
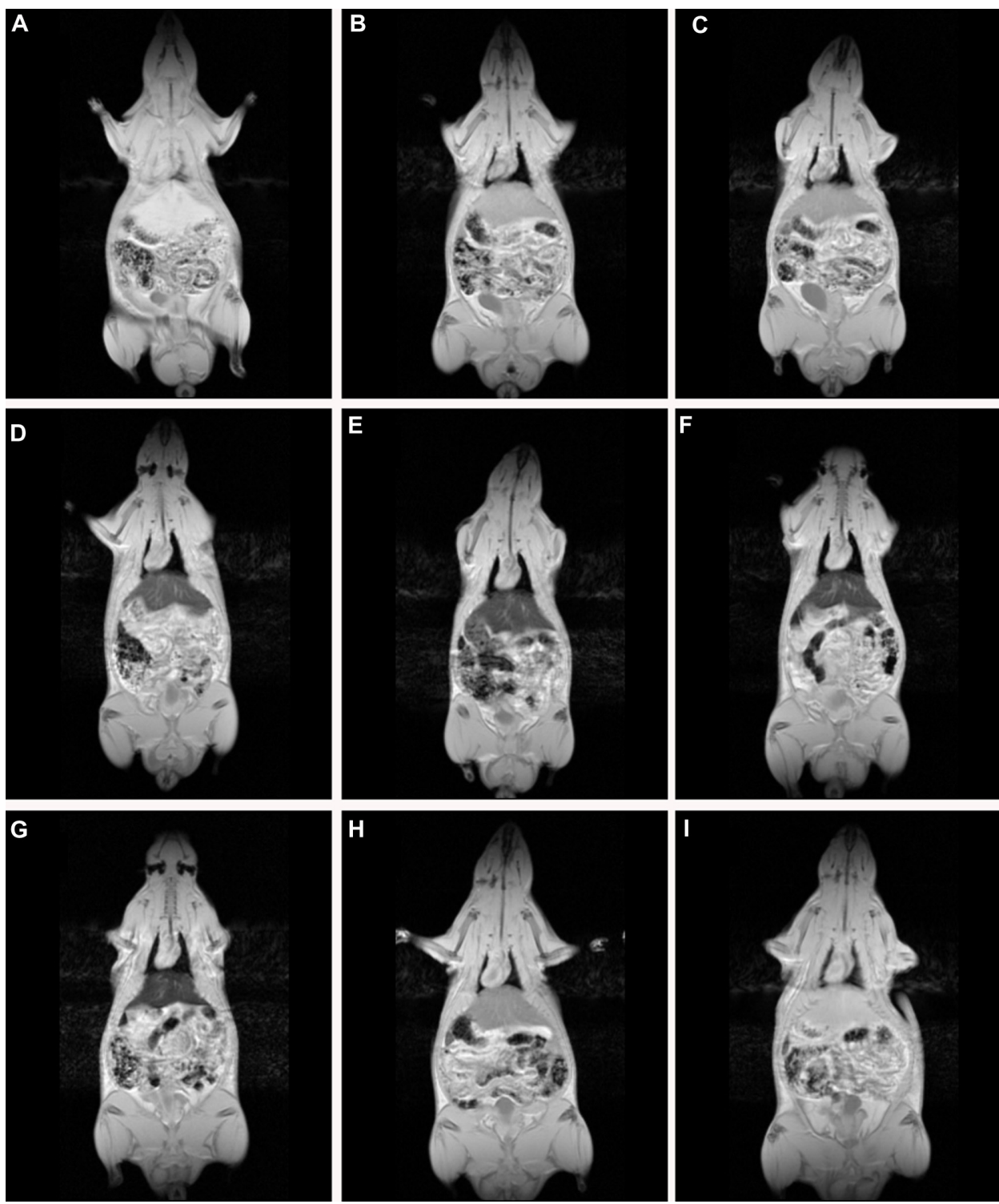

Figure 4 Dynamic magnetic resonance imaging of a rat after injection of SPION microbubbles. Magnetic resonance signal intensity changed mostly in the liver over time from 10 minutes to 6 weeks compared with pre-injection. (A) Pre-injection and at (B) 10 minutes, (C) one hour, (D) 24 hours, (E) 48 hours, (F) one week, (G) 2 weeks, (H) 4 weeks, and (I) 6 weeks post injection.

Abbreviation: SPION, superparamagnetic iron oxide. 


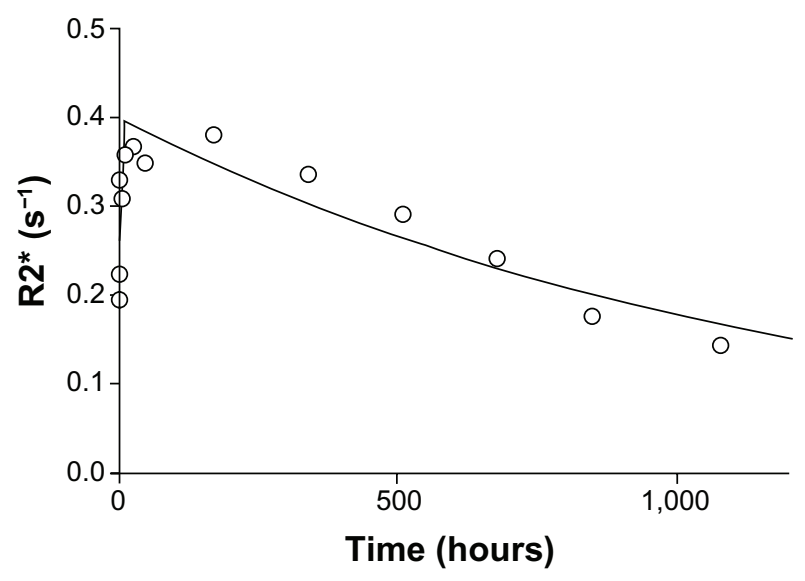

Figure 5 Pharmacokinetic model of SPION microbubbles. Relaxation times (R2*; s ${ }^{-1}$ ) of SPION microbubbles in the liver (as indicator for concentrations) versus time (hours) were fitted to a one-compartment open model. The elimination half-life was calculated from the slope.

Abbreviation: SPION, superparamagnetic iron oxide.

nodes, sternal bone marrow, and eyes) showed rare solitary microbubbles apparently free in the circulatory system from 10 minutes post injection onwards.

Clusters or aggregates of SPION microbubbles were largest and most frequent in the lungs after intravenous injection. At 10 minutes post injection (Figure 6A), numerous microbubbles were seen in the capillaries of the alveolar septa and less frequently in the occasionally distended small arterioles along the bronchial tree. Intravascular clusters are often associated with intravascular, finely fibrillar, and eosinophilic material (consistent with fibrin). Marked infiltration of inflammatory cells was absent in all organs investigated except for the lungs where, from 3 hours after injection, increased numbers of intravascular and rarely alveolar neutrophils were noted, as well as a minimal increase in macrophages associated with the clustered SPION microbubbles. Pulmonary macrophages became more apparent after 6 hours and neutrophil numbers further increased to a maximum at 12 hours post injection, after which neutrophil numbers decreased while macrophages remained and increased in size. Numbers of freely circulating microbubbles decreased from 24-48 hours post injection (Figure 6B). Neutrophil numbers further declined one week after injection and reached background levels at 2 weeks following injection (Figure 6C). From 48 hours after injection and later, the remaining clusters of SPION microbubbles associated with macrophages decreased in size. A decreased number of phagocytosed microbubbles were observed at 2 weeks (Figure 6C), while at 4 weeks following injection (Figure $6 \mathrm{D})$ the now abundant macrophage cytoplasm started to

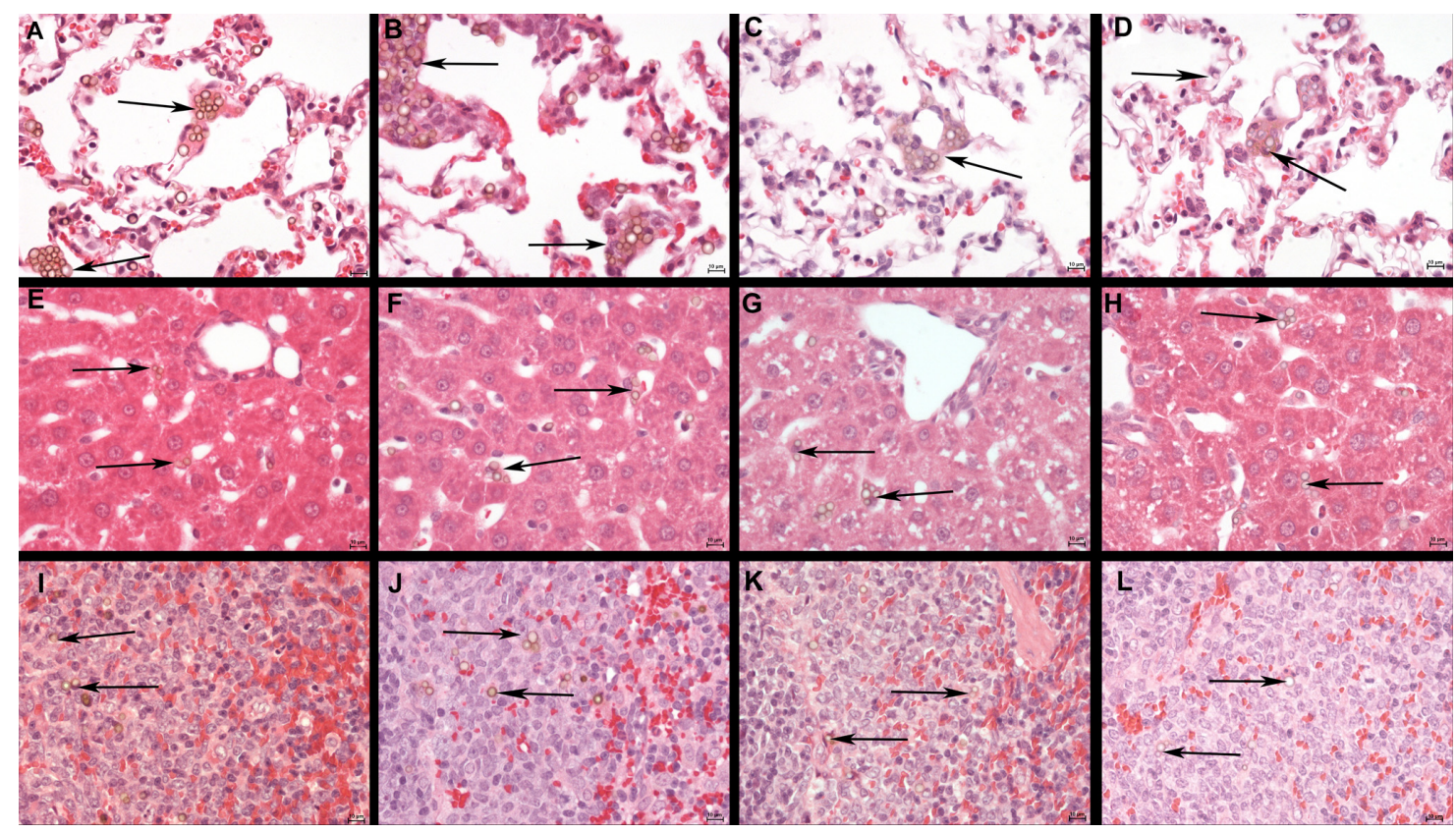

Figure 6 Histology of the lungs, liver, and spleen of a rat post injection of SPION microbubbles. Abundant intravascular, fibrin-covered microbubbles in lung at 10 minutes (A) and 24 hours (B). A decreased number of phagocytosed microbubbles were observed at 2 weeks (C). Iron pigment becomes apparent in macrophage cytoplasm after 4 weeks (D). In the liver, rare microbubbles are found in the vicinity of Kupffer cells at 10 minutes post injection (E), and increasingly phagocytosed at 24 hours (F). Phagocytosed microbubbles were abundant at 2 weeks post injection $(\mathbf{G})$ and decreased 4 weeks after injection $(\mathbf{H})$. In the spleen, 10 minutes after injection, microbubbles are found in red pulp and marginal zone (I). From 24 hours after injection and onwards, the microbubbles are associated with marginal zone macrophages and rare in red pulp (J). (K) At 2 weeks and (L) at 4 weeks. The size bar represents $10 \mu \mathrm{m}$. The arrows show the microbubbles in the different tissues.

Abbreviation: SPION, superparamagnetic iron oxide. 
show a brown color, indicative of accumulation of iron released from SPION microbubbles.

In the liver, small clusters of microbubbles (1-3) were found in the sinusoids as early as 10 minutes post injection, mostly associated with macrophages (Figure 6E). Microbubble clusters were more frequent in the periportal areas. Over the following hours, cluster frequency and size (6-8 microbubbles per Kupffer cell) increased (Figure 6F). Microbubble clusters became clearly less frequent at 2 weeks post injection (Figure 6G), whereas cluster size decreased more slowly but recognizably from 3 weeks post injection (Figure 6H). In the spleen, frequent microbubbles were observed from 10 minutes post injection, and seen predominantly as solitary microbubbles and small clusters (2-3 per cell) associated with macrophages in the marginal zone (Figure 6I). Fewer solitary bubbles were observed in the red pulp (Figure 6I). The frequency of microbubbles in the marginal zone peaked at 12 and 24 hours post injection (Figure 6J), whereas a mild decrease was observed in the red pulp as early as 24 hours post injection (Figure 6J). A negligible number of solitary bubbles was occasionally observed in the periarteriolar lymphoid sheaths from 3 weeks post injection. The marginal zone was not markedly expanded at any time point following injection. In the spleen, macrophages showed signs of cytoplasmic iron accumulation from 2 weeks post injection (Figure 6K). In the liver, these signs were not clearly visible on hematoxylin and eosin or Perl's staining at any time point investigated. Macrophages in the red pulp of the spleen showed massive accumulation of brown pigment (Figure 6L), consistent with iron accumulation, at 6 weeks post injection (Figure 7I).

We further investigated the pattern of SPION microbubble distribution in different tissues using Perl's Prussian blue staining for the detection of iron. Consistent with the hematoxylin and eosin staining results, single or clustered microbubbles were detected in lung, liver, and spleen as early as 10 minutes following administration of SPION microbubbles (Figure 7A, D, and G, respectively). Predominantly clustered microbubbles were seen in pulmonary macrophages at one week post injection (Figure 7B), and numbers decreased further until the latest time point analyzed (6 weeks post injection, Figure 7C). Perl's Prussian blue staining of SPION microbubbles in liver

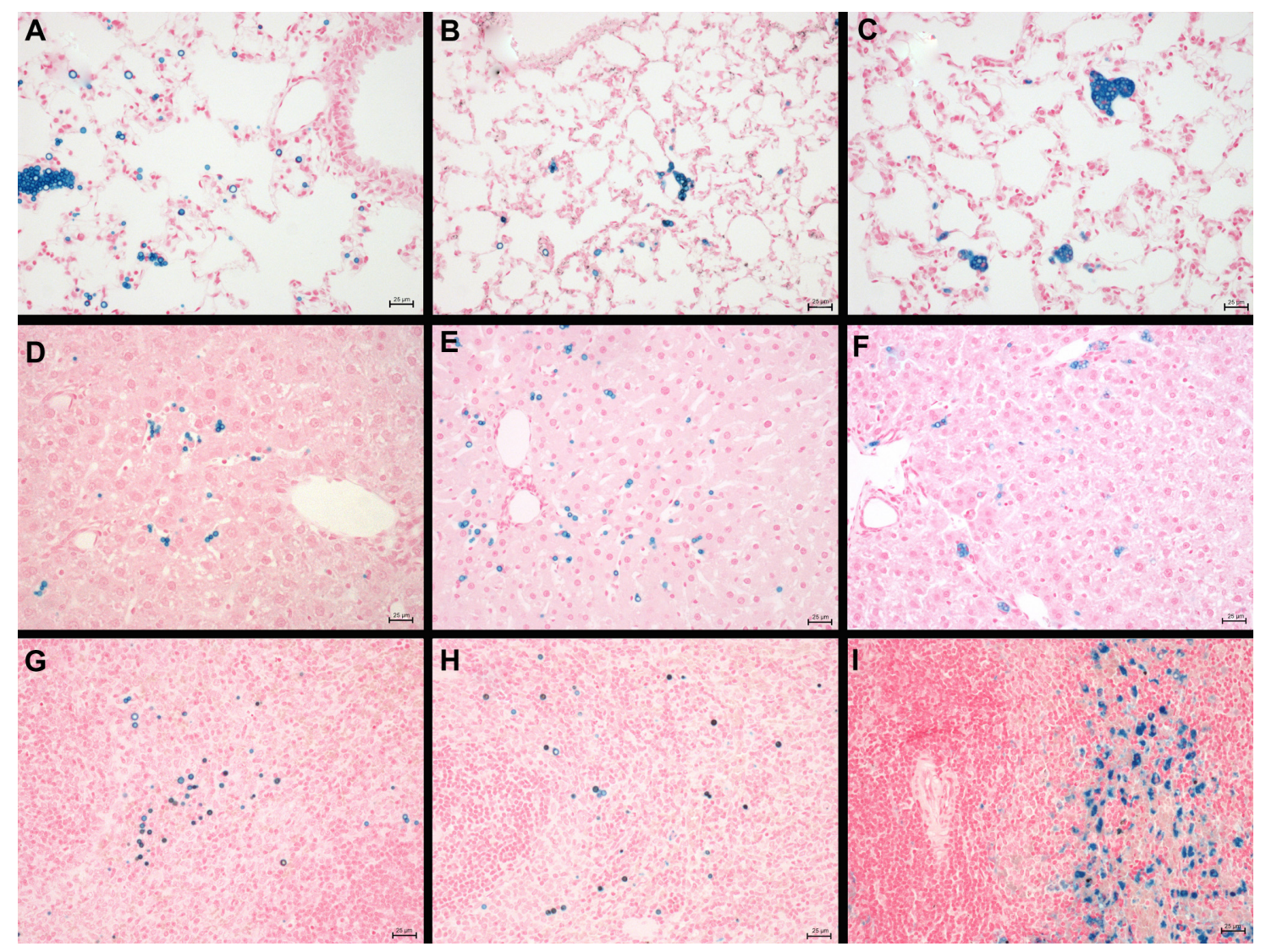

Figure 7 Distribution of iron after injection of SPION microbubbles in rat lungs, liver, and spleen at various time points visualized by Perl's Prussian blue staining. SPION microbubbles/iron at 10 minutes, one week, and 6 weeks post injection in (A-C) the lungs, (D-F) liver, and (G-I) spleen. The size bar represents $25 \mu \mathrm{m}$. Abbreviation: SPION, superparamagnetic iron oxide. 
tissue is shown in Figure 7D-F. Microbubbles were rarely found in the liver (Figure 7D) by 10 minutes post injection; however, a marked increase in their numbers was observed at one week post injection (Figure 7E). At 6 weeks post injection, the number of microbubbles in liver tissue declined (Figure 7F). Images of the spleen showed a number of single SPION microbubbles in the marginal zone at 10 minutes and at one week post injection (Figure $7 \mathrm{G}$ and $\mathrm{H}$ ) as well as at 6 weeks post injection (Figure 7I). Further, the number of single or clustered microbubbles was decreased but the iron content of the spleen was increased. The results of digital quantification of Perl's Prussian blue-stained microbubbles in the lungs, liver, and spleen over time are shown in Figure 8A-C. The maximum number of microbubbles in the lungs was detected at 10 minutes post injection. The number of SPION microbubbles was relatively low at 10 minutes post injection in both the liver and spleen compared with the lungs. The numbers then increased rapidly in the spleen to reach a maximum at 24 hours to one week post injection, whereas a more gradual increase was observed in the liver, resulting in a peak at two weeks post injection. At later time points (6 weeks), physiologic accumulation of iron in the splenic red pulp interfered with accurate digital assessment of microbubbles in the spleen. However, the amount of splenic iron in a similarly aged control rat was lower than the amount found in rats 6 weeks after injection (Figure 9).

Consistent with the hematoxylin and eosin staining results, Perl's Prussian blue staining showed a considerable amount of cytoplasmic iron in pulmonary macrophages at 2 weeks post injection. In addition, Perl's Prussian blue staining showed a minimal loss of iron to the macrophage cytoplasm as early as one week after administration. No increase in tissue iron content was noted outside the SPION microbubbles in rats repeatedly exposed to MR scans over one week, indicating that the SPION particles do not noticeably affect the structural integrity of the PVA in microbubbles in a 3 Tesla magnetic field. The role of macrophages in localizing the SPION microbubbles inside the tissue was further investigated using a macrophagespecific antibody and the intrinsic fluorescence property of the fluorescein-labeled SPION microbubbles in this study. Figure 10 demonstrates the intracellular localization of fluorescent SPION microbubbles inside labeled macrophages, which is consistent with the overall histology results and observed tissue distribution.

Apart from a single fibrin-coated microbubble attached to the sinusoid wall in a liver sample at 3 weeks post injection, no indications of hepatic thrombosis were observed
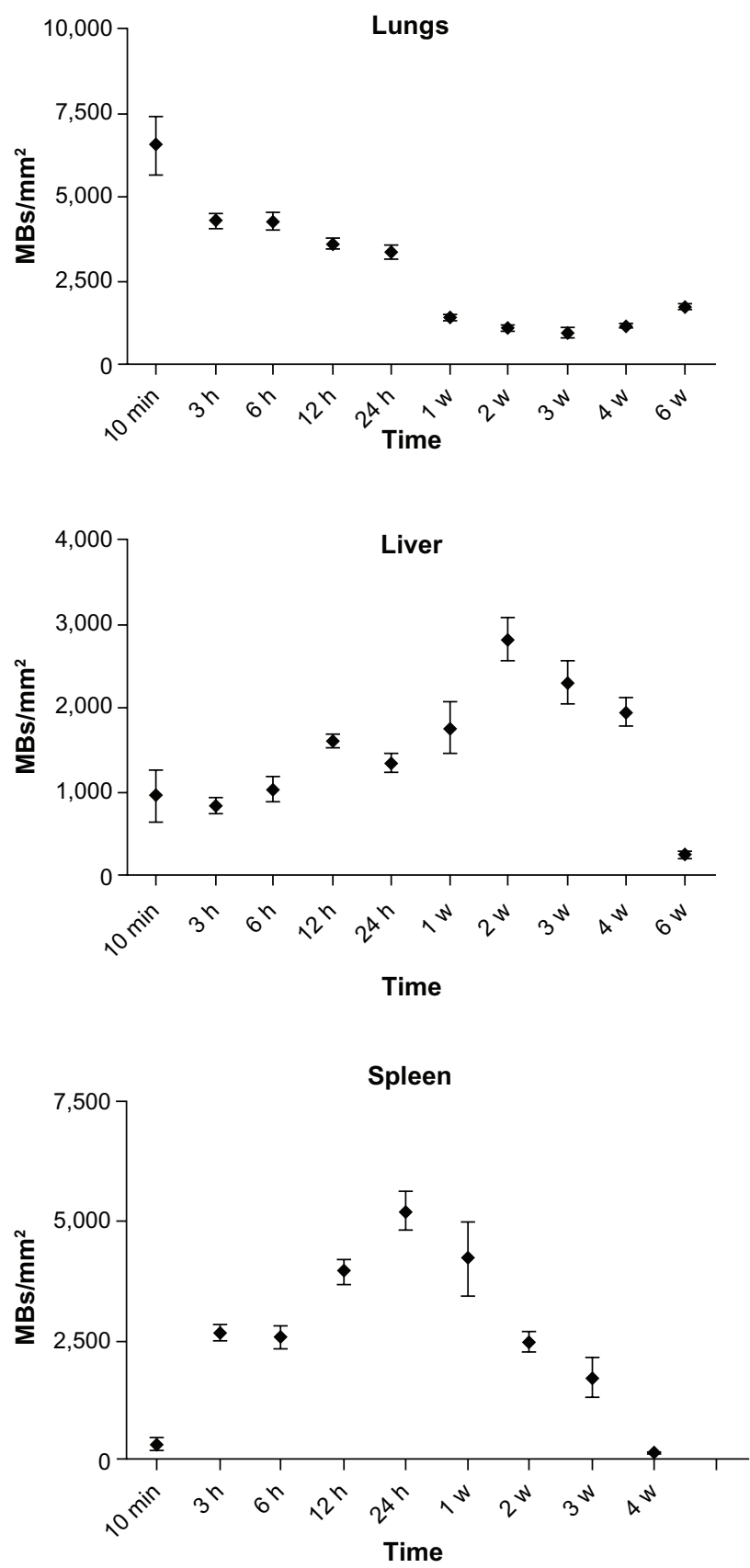

Figure 8 Digital quantification of the number of Perl's Prussian blue-stained SPION microbubbles in the lungs, liver, and spleen over time.

Abbreviations: SPION, superparamagnetic iron oxide; MBs, microbubbles; mins, minutes; h, hours; w, weeks.

and there was no increase in hepatic neutrophil numbers. A rare larger cluster of macrophages $(50 \mu \mathrm{m}$ in diameter $)$ associated with a few microbubbles was observed in one animal 6 weeks post injection, but otherwise no indications of protracted hepatic damage were observed. Intravascular mural thrombi containing SPION microbubbles, neutrophils, and occasional trapped erythrocytes and macrophages, as well as early signs of organization, were observed in the lungs of a limited number of animals 

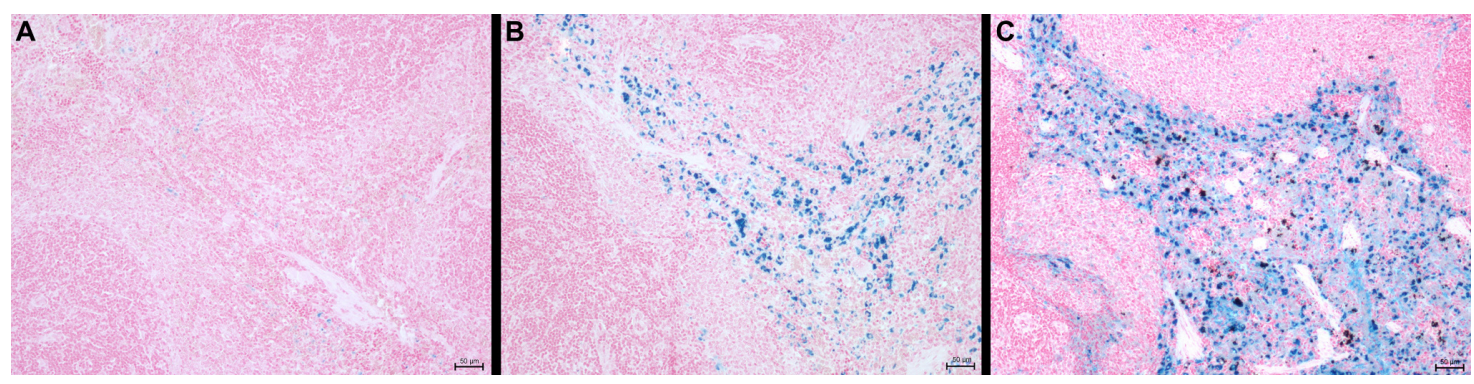

Figure 9 The amount of iron in the spleen of I2-week-old and 25-week-old control rats compared with a 25-week-old rat injected with SPION microbubbles as visualized using Perl's Prussian blue staining. (A) The spleen of a 12-week-old control rat (untreated) shows a limited amount of iron, while (B) in the 25-week-old control rat, the amount of iron was increased. (C) Shows the amount of iron found in the spleen of the 25 -week-old rat injected 6 weeks previously with SPION microbubbles, that was considerably higher compared with the untreated control.

Abbreviation: SPION, superparamagnetic iron oxide.

(Figure 11A) at 6 and 12 hours. Thrombi were no longer observed after 3 weeks and onwards; however, one animal sacrificed at 3 weeks and another at 6 weeks showed larger clusters of macrophages with occasional neutrophils and proliferation of bronchus-associated lymphoid tissue (Figure 11B), indicating granulomatous pneumonia and a possible risk of mild, longer persisting pulmonary damage at the currently used dose.

Marked presence of SPION microbubbles was not observed in any of the other organs investigated microscopically. The kidneys showed occasional solitary microbubbles in the glomerular capillaries (Figure 11C),
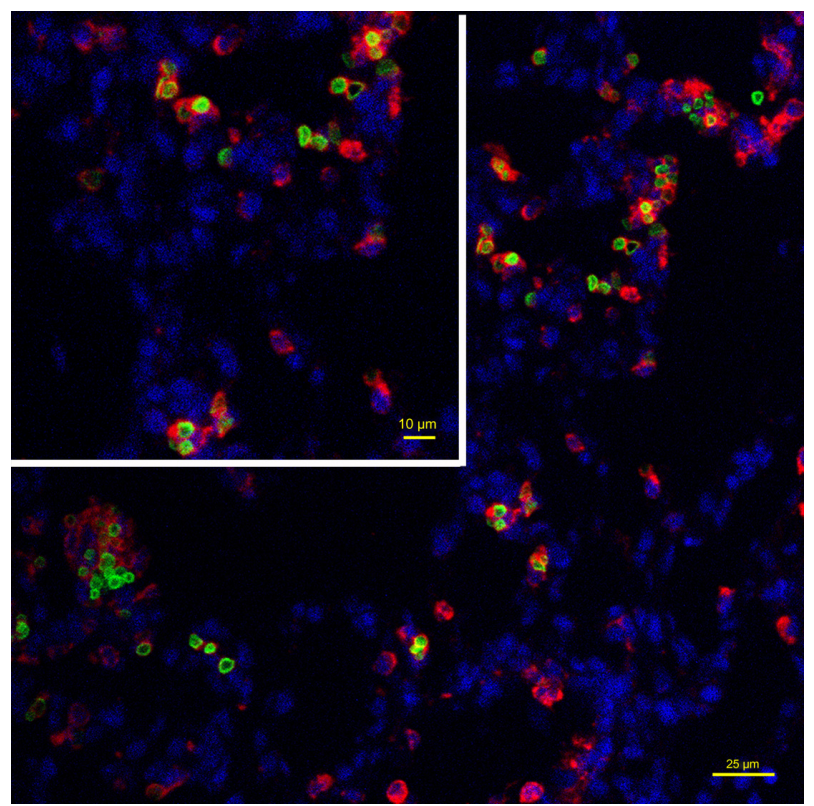

Figure 10 Intracellular localization of fluorescent SPION microbubbles inside labeled macrophages. A primary antibody against CD68 (red) confirms phagocytosis of the microbubbles (green) by macrophages in the lungs. The nuclei were stained blue using DAPI. The size bar represents $25 \mu \mathrm{m}$. The upper left part of the image is higher magnification with the size bar of $10 \mu \mathrm{m}$.

Abbreviations: DAPI, 4',6-diamidino-2-phenylindole; SPION, superparamagnetic iron oxide. increasing up to 6 hours post injection and thereafter gradually decreasing until becoming absent from 4 weeks post injection. Microbubbles were not observed in the mesangium. Rare microbubbles were observed in the cortical interstitial blood vessels until one week post injection. Occasional mild tubular protein was observed, but not in relation to glomerular microbubble frequency. However, this observation was slightly more frequent around 6 and 12 hours post injection, coinciding with the influx of pulmonary neutrophils, which might indicate a mild cytokinerelated proteinuria at these time points.

Bone marrow showed infrequent solitary microbubbles, sometimes phagocytosed but also free in the sinuses. The bone marrow showed no other abnormalities, but gradually increasing nuclear hypertrophy was recognized in endosteal cells from 3 weeks onwards. At 6 weeks post injection, some deposition of osteoid was observed along bone trabeculae, with a slight brown discoloration of recently deposited subendosteal lamellar bone (data not shown).

Brain and eye tissue showed rare, mostly free, intravascular bubbles until 48 hours post injection, not related to brain regions and located in the choroid capillaries of the eye. Apart from negligible single microbubbles in the cortex of mesenteric lymph nodes in three animals at different time points across the study, no microbubbles were observed in the lymph nodes collected.

\section{Discussion}

Recent advances in nanoscience and the development of nanoparticles have revolutionized biomedicine during the last decade. The characteristics of engineered nanoparticles can be easily manipulated for use in drug delivery and various routes for tracing, administration, and controlled release. Microbubbles with a PVA matrix can also be tailored as an imaging agent or drug carrier. In the present study, we 


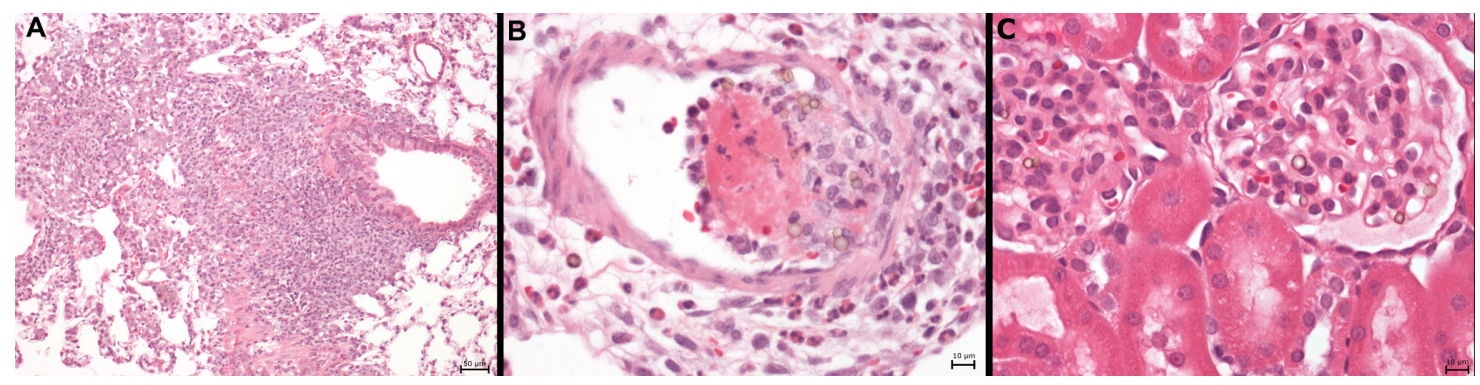

Figure II Protracted pulmonary pathology and example of glomerular SPION microbubbles. (A) Macrophages engulfing SPION microbubbles in a mural intravascular thrombus associated with neutrophils in the lung of one rat 24 hours post injection and (B) localized chronic inflammation characterized by accumulated macrophages, fewer neutrophils, and marked fibrosis in the lung of a rat, 4 weeks post injection of SPION microbubbles. (C) Renal cortex showing occasional SPION microbubbles in the glomerular vascular tuft. Size bars represent 50, 10, and $10 \mu \mathrm{m}$, respectively.

Abbreviation: SPION, superparamagnetic iron oxide.

investigated the biodistribution of a novel microdevice, ie, SPION incorporating microbubbles, by using and comparing the results from 3 Tesla MR imaging with histopathologic findings. Previously, using SPECT/MR fusion imaging, we have shown that SPION microbubbles are rapidly located to the lungs (10 minutes, SPECT) and accumulated in the liver from 2 to 24 hours post injection (SPECT/MR imaging). ${ }^{23}$ Recently, elimination of single-layer SPION microbubbles from the liver has been studied by quantitative signal decrease measurements and $\mathrm{T} 2 *$ calculations from 3 Tesla MR images, and a half-life of 25 days was calculated. In the present MR study, the calculated half-life of multilayer SPION microbubbles was slightly longer ( $\mathrm{t}_{1 / 2}, 31$ days) compared with that calculated in our previous study. This could be due to several factors, including the multiple layers used in the current SPION microbubbles, causing them to degrade and/or release iron more slowly. Comparing the effect of different formulations of iron oxide on the biological halflife in rat tissue, Briley-Saebo et al found that the half-life of iron oxide nanoparticles coated with oxidized starch was 29 days. The authors reported biphasic elimination with an initial half-life of 8 days followed by a slower elimination phase of 43-46 days in rat liver. ${ }^{5,29}$ Briley-Saebo et al also reported that the MR signal in the liver returns to normal values 4 weeks after administration of dextran-coated iron oxide nanoparticles, which is sooner than that found in the current study (6 weeks).

Moreover, using oxidized starch-coated iron oxide, the authors reported increased traces of iron in hepatic Kupffer cells from 18 days post injection of iron oxide nanoparticles, as examined microscopically. ${ }^{5}$ Our present results show accumulation of free iron in the cytoplasm of pulmonary macrophages at 2 weeks post injection. However, this was not observed in the liver. In the spleen, marked presence of iron pigment became apparent only after 6 weeks and predominantly in the red pulp, coinciding with the natural iron increase occurring in the spleen of rats on a standard diet. Keeping in mind the rapid, initial decrease of iron oxide reported for oxidized starch-coated nanoparticles, ${ }^{5}$ it is therefore conceivable that the PVA shell and the larger particle size of SPION microbubbles causes their slower degradation in macrophages..$^{30}$ These results indicate more stable iron loading into the administered SPION microbubbles. Using SPION incorporated into microbubbles as a vehicle for drug delivery might therefore result in more sustained release, and this should be investigated further. Various other formulations of iron oxide have been proposed, including polymeric immunomicelles, ${ }^{31}$ one-layer microbubbles, dextranencapsulated nanoparticle clusters, dextran-coated iron oxide, ${ }^{5}$ poly-L-lysine hydrobromide-coated SPION, ${ }^{32}$ polysaccharide surface-modified SPION ${ }^{33}$ dextran-encapsulated nanoparticle clusters, ${ }^{34}$ and starch-coated SPION $^{35}$ for contrast enhancement in medical and investigational imaging. Munnier et al have successfully utilized SPION as a magnetic traceable agent for delivery of doxorubicin..$^{10}$ Rappeport et al reported that SPIONbased contrast agents, such as ferumoxides and ferucarbotrans, are specifically used for spleen and liver MR imaging because these agents are taken up by both macrophages in the spleen and Kupffer cells in the liver. ${ }^{36,37}$ In the present study, we also observed accumulation of iron in both organs.

The presently used SPION microbubbles have a narrow size range and are well dispersed, which facilitates intravenous application. Polymer-based nanoparticles conjugated to fluorescent markers and targeting antibodies have been used with fewer reported toxic side effects compared to those derived from biological systems. ${ }^{2}$ Loading of SPION into other constructed particles, such as liposomes, may modify particle size and hence affect biodistribution and elimination of the contrast agent and the drug delivery device. ${ }^{38}$ Large gas-filled microbubbles provide additional ultrasound contrast. ${ }^{39,40}$ The present investigation addresses how larger 
vehicles would affect the distribution and half-life of the iron oxide signal using MR imaging. Microscopic tissue sample analysis confirmed rapid uptake of SPION microbubbles in the lungs and their redistribution to the liver and spleen over time. As we have previously hypothesized, and in line with findings of various authors regarding smaller intravenously administered nanoparticles, ${ }^{3,41-43}$ histology showed the prominent role of macrophage uptake along the pulmonary capillary bed and (predominantly periportal) hepatic sinusoids. Further, these results were confirmed utilizing immunohistochemistry. Despite distribution to the liver and the relatively long half-life of SPION microbubbles, we did not observe signs of necrosis, inflammation, or fibrosis in the liver and spleen. These findings are in agreement with those of Bacon et al, ${ }^{45}$ who studied several biochemical liver parameters for redox toxicity (including hepatic, mitochondrial, and microsomal lipid peroxidation) in rats after intravenous ferrite injections, without finding evidence of iron-induced hepatotoxicity or organelle dysfunction. Therefore, it is unlikely that an increased iron load after injection of SPION microbubbles will give rise to adverse effects. The loss of iron from SPION microbubbles in the present study occurred gradually, and had already appeared while the morphological integrity was still preserved. A marked increase in iron load in the spleen was finally observed when frequent signs of integrity loss were seen at 6 weeks post administration. Additional evaluation of how repeated application of a 3 Tesla magnetic field might affect integrity of the microbubble wall and accelerate iron loss to the tissues did not show any histologic indication for increased tissue iron load. The initially widespread intravascular clustering of SPION microbubbles in the lungs largely resolved after 3 weeks; however, an indication of possible adverse effects resulting from intravenous administration of large numbers of SPION microbubbles was found in the lungs of two rats at later time points, where we observed remains of organized vascular thrombi and focal granulomas. In these rats, localized granulomas had formed in association with unresolved aggregates of microbubbles. Further studies addressing the mode, speed of administration, and biological fate of SPION microbubbles should clarify if these potentially negative effects can be attenuated and whether slower degradation of these particles can serve in a complementary role to existing nanoscale targeted drug delivery systems.

\section{Conclusion}

Histologic findings are generally correlated with MR imaging. The methods used in the current study appropriately characterize the properties of SPION microbubbles in terms of size, size distribution, the effect on rat tissue in various organs in vivo, cellular distribution, biological fate, and the relaxivity of SPION microbubbles using MR imaging. Further investigations should address the dynamics of drug release from the microbubbles, modifications for organ targeting, size effect on biodistribution and kinetics, tendency to cluster in vivo, and delivery dynamics during administration to attenuate possible adverse effects on the pulmonary vasculature.

\section{Acknowledgment}

This work was supported by the European Commission project 3MICRON within the framework of the Seventh Framework Program, the Swedish Cancer Foundation (CancerFonden), and the Swedish Children's Cancer Society (BarnCancerFonden).

\section{Disclosure}

The authors declare that they have no conflict of interest in this work.

\section{References}

1. Semete B, Booysen L, Lemmer Y, et al. In vivo evaluation of the biodistribution and safety of PLGA nanoparticles as drug delivery systems. Nanomedicine. 2010;6(5):662-671.

2. Singh P, Prasuhn D, Yeh RM, et al. Bio-distribution, toxicity and pathology of cowpea mosaic virus nanoparticles in vivo. $J$ Control Release. 2007;120(1-2):41-50.

3. Xie G, Sun J, Zhong G, Shi L, Zhang D. Biodistribution and toxicity of intravenously administered silica nanoparticles in mice. Arch Toxicol. 2010;84(3):183-190.

4. Yoon HY, Saravanakumar G, Heo R, et al. Hydrotropic magnetic micelles for combined magnetic resonance imaging and cancer therapy. J Control Release. 2012;160(3):692-698.

5. Briley-Saebo K, Hustvedt SO, Haldorsen A, Bjornerud A. Longterm imaging effects in rat liver after a single injection of an iron oxide nanoparticle based MR contrast agent. J Magn Reson Imaging. 2004;20(4):622-631.

6. Inoh K, Muramatsu H, Torii S, et al. Doxorubicin-conjugated antimidkine monoclonal antibody as a potential anti-tumor drug. Jpn $J$ Clin Oncol. 2006;36(4):207-211.

7. Mahmoudi M, Sant S, Wang B, Laurent S, Sen T. Superparamagnetic iron oxide nanoparticles (SPIONs): development, surface modification and applications in chemotherapy. Adv Drug Deliv Rev. 2011;63(1-2): $24-46$.

8. Kim do K, Chang JH, Kang YJ. Efficient internalization of peptideconjugated SPIONs in dendritic cells for tumor targeting. J Nanosci Nanotechnol. 2012;12(7):5191-5198.

9. Vingerhoeds MH, Haisma HJ, van Muijen M, van de Rijt RB, Crommelin DJ, Storm G. A new application for liposomes in cancer therapy. Immunoliposomes bearing enzymes (immuno-enzymosomes) for site-specific activation of prodrugs. FEBS Lett. 1993;336(3): 485-490.

10. Munnier E, Cohen-Jonathan S, Linassier C, et al. Novel method of doxorubicin-SPION reversible association for magnetic drug targeting. Int J Pharm. 2008;363(1-2):170-176.

11. Laroui H, Sitaraman SV, Merlin D. Gastrointestinal delivery of antiinflammatory nanoparticles. Methods Enzymol. 2012;509:101-125. 
12. Wang J, Wang X, Song Y, Zhu C, Wang K, Guo Z. Detecting and delivering platinum anticancer drugs using fluorescent maghemite nanoparticles. Chem Commun (Camb). 2013;49(27):2786-2788.

13. Carney CE, Tran AD, Wang J, Schabel MC, Sherry AD, Woods M. Towards the rational design of MRI contrast agents: delta-substitution of lanthanide(III) NB-DOTA-tetraamide chelates influences but does not control coordination geometry. Chemistry. 2011;17(37): 10372-10378.

14. Martinez GV, Zhang X, Garcia-Martin ML, et al. Imaging the extracellular $\mathrm{pH}$ of tumors by MRI after injection of a single cocktail of T1 and T2 contrast agents. NMR Biomed. 2011;24(10): 1380-1391.

15. Wu Y, Carney CE, Denton M, et al. Polymeric PARACEST MRI contrast agents as potential reporters for gene therapy. Org Biomol Chem. 2010;8(23):5333-5338.

16. Wu Y, Zhou Y, Ouari O, et al. Polymeric PARACEST agents for enhancing MRI contrast sensitivity. J Am Chem Soc. 2008;130(42): 13854-13855.

17. Borel A, Bean JF, Clarkson RB, et al. Towards the rational design of MRI contrast agents: electron spin relaxation is largely unaffected by the coordination geometry of gadolinium(III)-DOTA-type complexes. Chemistry. 2008;14(9):2658-2667.

18. Woods M, Botta M, Avedano S, Wang J, Sherry AD. Towards the rational design of MRI contrast agents: a practical approach to the synthesis of gadolinium complexes that exhibit optimal water exchange. Dalton Trans. 2005;24:3829-3837.

19. Lin SP, Brown JJ. MR contrast agents: physical and pharmacologic basics. J Magn Reson Imaging. 2007;25(5):884-899.

20. Frullano L, Catana C, Benner T, Sherry AD, Caravan P. Bimodal MR-PET agent for quantitative $\mathrm{pH}$ imaging. Angew Chem Int Ed Engl. 2010;49(13):2382-2384.

21. Zhang B, Li Q, Yin P, et al. Ultrasound-triggered BSA/SPION hybrid nanoclusters for liver-specific magnetic resonance imaging. ACS Appl Mater Interfaces. 2012;4(12):6479-6486.

22. Yoo MK, Park IK, Lim HT, et al. Folate-PEG-superparamagnetic iron oxide nanoparticles for lung cancer imaging. Acta Biomater. 2012;8(8):3005-3013.

23. Barrefelt AA, Brismar TB, Egri G, et al. Multimodality imaging using SPECT/CT and MRI and ligand functionalized 99mTc-labeled magnetic microbubbles. EJNMMI Res. 2013;3(1):12.

24. Wells DJ. Animal welfare and the 3 Rs in European biomedical research. Ann N Y Acad Sci. 2011;1245:14-16.

25. Madden JC, Hewitt M, Przybylak K, Vandebriel RJ, Piersma AH, Cronin MT. Strategies for the optimisation of in vivo experiments in accordance with the 3Rs philosophy. Regul Toxicol Pharmacol. 2012;63(1):140-154.

26. Schiffelers MJ, Blaauboer BJ, Hendriksen CF, Bakker WE. Regulatory acceptance and use of 3R models: a multilevel perspective. ALTEX. 2012;29(3):287-300.

27. Peyratout CS, Dahne L. Tailor-made polyelectrolyte microcapsules: from multilayers to smart containers. Angew Chem Int Ed Engl. 2004;43(29):3762-3783.

28. Morawietz G, Ruehl-Fehlert C, Kittel B, et al. Revised guides for organ sampling and trimming in rats and mice. Part 3. A joint publication of the RITA and NACAD groups. Exp Toxicol Pathol. 2004;55(6): 433-449.
29. Briley-Saebo KC, Johansson LO, Hustvedt SO, et al. Clearance of iron oxide particles in rat liver: effect of hydrated particle size and coating material on liver metabolism. Invest Radiol. 2006;41(7): $560-571$.

30. Shi $\mathrm{R}, \mathrm{Zhu} \mathrm{AC}$, Chen $\mathrm{DF}$, et al. In vitro degradation of starch/PVA films and biocompatibility evaluation. J Appl Polym Sci. 2010;115(1): 346-357.

31. Sawant RM, Sawant RR, Gultepe E, et al. Nanosized cancer cell-targeted polymeric immunomicelles loaded with superparamagnetic iron oxide nanoparticles. J Nanopart Res. 2009:1777-1785.

32. Albukhaty S, Naderi-Manesh $H$, Tiraihi T. In vitro labeling of neural stem cells with poly-L-lysine coated super paramagnetic nanoparticles for green fluorescent protein transfection. Iran Biomed J. 2013;17(2): 71-76

33. Zhu A, Yuan L, Jin W, et al. Polysaccharide surface modified $\mathrm{Fe}_{3} \mathrm{O}_{4}$ nanoparticles for camptothecin loading and release. Acta Biomater. 2009;5(5):1489-1498.

34. Mondalek FG, Zhang YY, Kropp B, et al. The permeability of SPION over an artificial three-layer membrane is enhanced by external magnetic field. J Nanobiotechnology. 2006;4:4.

35. Kim DK, Mikhaylova M, Wang FH, et al. Starch-coated superparamagnetic nanoparticles as MR contrast agents. Chem Mater. 2003;15(23): 4343-4351.

36. Rappeport ED, Loft A. Liver metastases from colorectal cancer: imaging with superparamagnetic iron oxide (SPIO)-enhanced MR imaging, computed tomography and positron emission tomography. Abdom Imaging. 2007;32(5):624-634.

37. Rappeport ED, Loft A, Berthelsen AK, et al. Contrast-enhanced FDG-PET/CT vs SPIO-enhanced MRI vs FDG-PET vs CT in patients with liver metastases from colorectal cancer: a prospective study with intraoperative confirmation. Acta Radiol. 2007;48(4):369-378.

38. Floris A, Ardu A, Musinu A, et al. SPION@liposomes hybrid nanoarchitectures with high density SPION association. Soft Matter. 2011;7(13):6239-6247.

39. Grishenkov D, Pecorari C, Brismar TB, Paradossi G. Characterization of acoustic properties of PVA-shelled ultrasound contrast agents: ultrasound-induced fracture (part II). Ultrasound Med Biol. 2009;35(7): 1139-1147.

40. Brismar TB, Grishenkov D, Gustafsson B, et al. Magnetite nanoparticles can be coupled to microbubbles to support multimodal imaging. Biomacromolecules. 2012;13(5):1390-1399.

41. Zahr AS, Davis CA, Pishko MV. Macrophage uptake of core-shell nanoparticles surface modified with poly(ethylene glycol). Langmuir. 2006;22(19):8178-8185.

42. Beduneau A, Ma Z, Grotepas CB, et al. Facilitated monocytemacrophage uptake and tissue distribution of superparmagnetic ironoxide nanoparticles. PloS One. 2009;4(2):e4343.

43. Nicolete R, dos Santos DF, Faccioli LH. The uptake of PLGA micro or nanoparticles by macrophages provokes distinct in vitro inflammatory response. Int Immunopharmacol. 2011;11(10):1557-1563.

44. Bacon BR, Stark DD, Park CH, et al. Ferrite particles: a new magnetic resonance imaging contrast agent. Lack of acute or chronic hepatotoxicity after intravenous administration. J Lab Clin Med. 1987; 110(2):164-171. 


\section{Publish your work in this journal}

The International Journal of Nanomedicine is an international, peerreviewed journal focusing on the application of nanotechnology in diagnostics, therapeutics, and drug delivery systems throughout the biomedical field. This journal is indexed on PubMed Central, MedLine, CAS, SciSearch $\AA$, Current Contents ${ }^{\circledR} /$ Clinical Medicine,

Journal Citation Reports/Science Edition, EMBase, Scopus and the Elsevier Bibliographic databases. The manuscript management system is completely online and includes a very quick and fair peer-review system, which is all easy to use. Visit http://www.dovepress.com/ testimonials.php to read real quotes from published authors.

Submit your manuscript here: http://www.dovepress.com/international-journal-of-nanomedicine-journal 